\title{
ANALYSING HYPER SPECTRAL REFLECTANCE WITH GAUSSIAN PROCESS AND COMBINED KERNEL
}

\author{
Sunil Singarapu ${ }^{1}$, Dr. Avinash Gour ${ }^{2}$ \\ ${ }^{1}$ Research Scholar, Dept. of Electronics and Communication Engineering, Sri Satya Sai University \\ of Technology \& Medical Sciences, Sehore, Bhopal-Indore Road, Madhya Pradesh, India. \\ ${ }^{2}$ Research Guide, Dept. of Electronics and Communication Engineering, Sri Satya Sai University of \\ Technology \& Medical Sciences, Sehore, Bhopal Indore Road, Madhya Pradesh, India \\ Email: sunil.sun0505@gmail.com

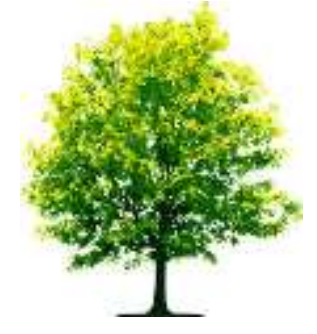 \\ Keywords: \\ Gaussian Process, Hyper \\ Spectral Image, Kernel, \\ Reflectance, Matrix.

\begin{abstract}
A B S T RA C T
The name speaks to that Gaussian cycle, subsequently we will utilize some reliable info datasets to test and prepare the hyper phantom picture. Piece based breaking down is a criticism framework used to contrast the assessed yield and the real yield. The distinction between those two yields will be considered as blunder and furthermore will be remedied at the information picture. The Gaussian Process is a powerful framework, so any blunder identified at yield will be assessed and prepared utilizing the Gaussian cycle and will be contrasted and the projection or anticipated network. In view of the correlation gave by the Gaussian cycle the framework will receives to revision for exact or wanted yield. Rectification speaks to the missing information, for example, shading, contrast, tone and so on the examination among proposed and real outcomes will demonstrate the effectiveness of ghostly investigation utilizing Gaussian cycle. This paper proposes a piece based hyper ghostly picture investigate utilizing Gaussian cycle to sort out the mistakes present at the framework yield.
\end{abstract}




\section{International Journal of}

Advanced Multidisciplinary Scientific Research (IJAMSR) ISSN:2581-4281

\section{Introduction}

Multispectral imaging, now and again implied as hyper-phantom imaging, has even more starting late become a critical instrument for confocal microscopy. This philosophy has for a long while been used in various fields, in any case, for instance, distant detecting, in which point by point pictures of scene and fundamental assortments of the world's surface are conveyed by separating different otherworldly signs made by scattered and reflected daylight. Assortments in ghostly information are constrained by assessing power contrasts at different frequencies from a comparable field of view, much like using a spectrometer to measure fluorescence from a cuvette at different frequencies. Picture instructive files are penniless down for individual ghastly frequencies and pixels with organizing unearthly information, as often as possible showing similar structures, are requested to take a picture. Since different scenes interface with light in a surprising manner, otherworldly assortments can be used to decide nuances in the field of view that can't be found in single pictures taken when all the light from the perceptible range is used or with single channels ${ }^{[1]}$.

\section{Hyperspectral Imaging}

Hyperspectral imaging is a system solidifying spectroscopy and imaging, where each picture is picked up at a flimsy band of the electromagnetic range. For example, the normal eye sees light in three gatherings (red, green and blue) of the undeniable range while hyperspectral imaging secludes the range in more gatherings, routinely covering the observable and close infrared reach. The term hyperspectral imaging suggests the relentless acquiring of tight gatherings $(<10 \mathrm{~nm})$ over the electromagnetic range. With our exceptional advancement we can get gatherings of $2 \mathrm{~nm}-4 \mathrm{~nm}$ wide and even $0.3 \mathrm{~nm}$. In fact, multispectral imaging covers simply a discrete number of gatherings, and is consistently performed with a channel wheel [2]. Through the examination of the unearthly and spatial information contained in each pixel of the picture, it is possible to perceive novel ghostly stamps and assign them to the pieces of the model under investigation. For example, the material or tissue dismembered can be arranged by its nuclear sections. Hyperspectral imaging has become dynamically standard throughout ongoing years in military, mechanical, and sensible fields. The ability to 


\section{International Journal of}

Advanced Multidisciplinary Scientific Research (IJAMSR) ISSN:2581-4281

unequivocally depict the shade of a saw thing, whether or not a hidden vehicle, an injury on an arm or on regular item, or a wide zone of vegetation, allows the customer to make taught decisions just yearned for previously. What once required colossal, delicate, and exorbitant exploration place spectrometers is by and by being done dynamically ready satellites, mechanized flying vehicles, and helpful handheld units. Hyperspectral imaging is the getting and handling of a picture at a very tremendous number of frequencies. Despite the fact that multispectral imaging may evaluate a picture in three or four shades (red, green, blue and close infrared (NIR), for example), hyperspectral imaging isolates the picture into tens or a few tints.

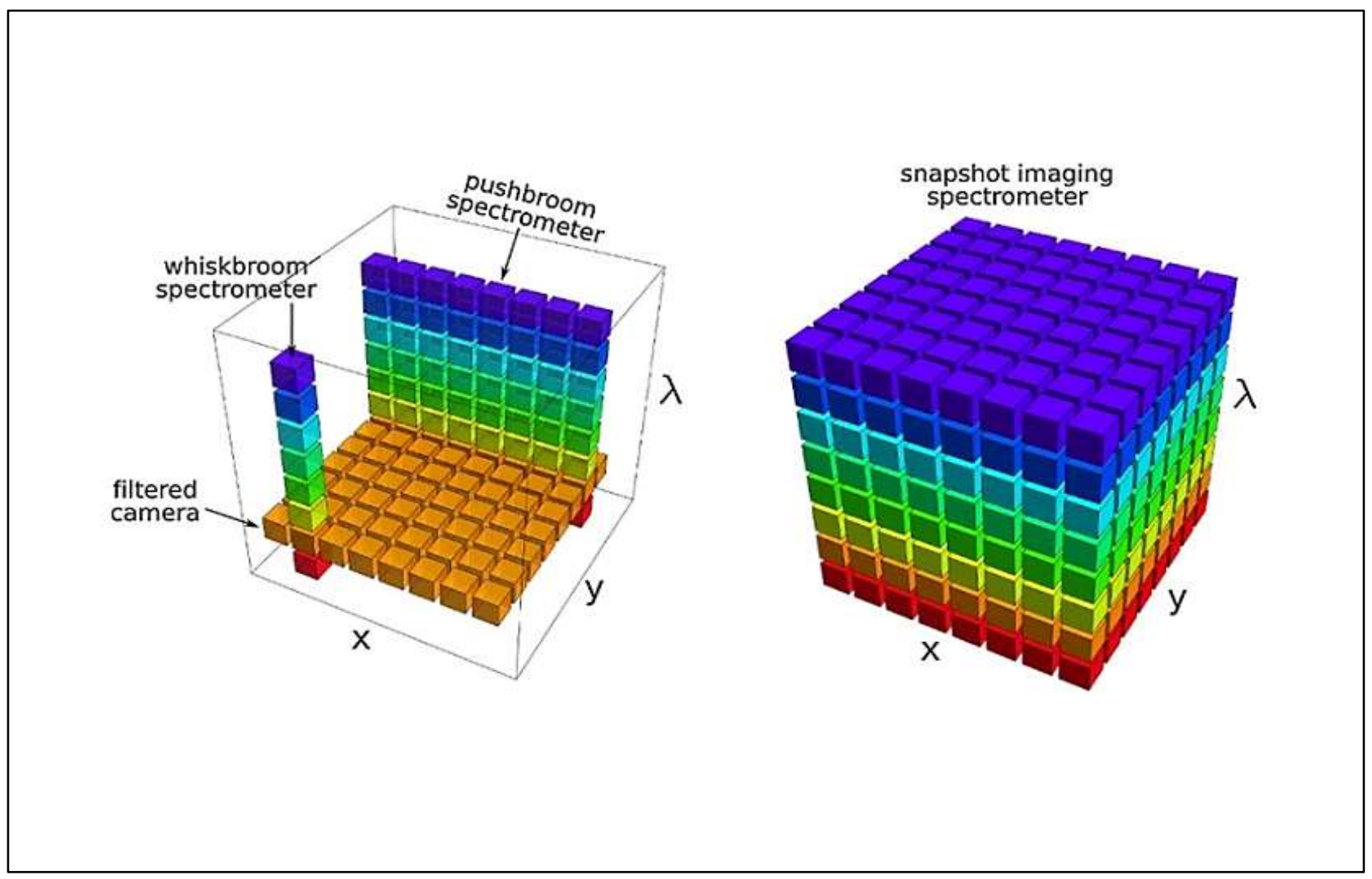

Figure 1 Hyperspectral Cubical Image 


\section{International Journal of}

Advanced Multidisciplinary Scientific Research (IJAMSR) ISSN:2581-4281

Extending a dream framework to make some more "containers" can be particularly muddled. In 1999, Deposition Sciences, Inc. (DSI) was contracted to cover a progression of 16 shaft splitters. These shaft splitters were designed in four essential optical chains, each with four auxiliary chains, bringing about sixteen discrete optical ways, each ending at a different locator. The final product was a sixteenchannel spectral imaging framework covering a frequency go from 400 - 900 nm. Each optical component needed to keep up a conveyance and reflectance of more prominent than $98 \%$ to guarantee a last sign level at the locators of at any rate $90 \%$. While this framework filled in as structured, it was enormous, costly, and fragile. It was reasonable for the benchtop show required, yet not for a considerable lot of the in-field applications required by the present sensor frameworks. The monochromatic images gained structure what we call a hyperspectral information shape, which contains both the spatial and spectral data of an example. In the hyperspectral 3D square, the initial two measurements are spatial (x,y pivot) while the third measurement ( $\mathrm{z}$ hub) is the frequency. Contingent upon the size of the sensor utilized, one single $3 \mathrm{D}$ shape can speak to numerous gigabytes of information speaking to an incredibly rich wellspring of data for material researchers or biomedical analysts [3][4].

\section{Gaussian Models}

Gaussian processes are an integral asset in the machine learning tool compartment. They permit us to make predictions about our information by fusing earlier information. Their most evident region of use is fitting a function to the information. This is called regression and is utilized, for instance, in mechanical autonomy or time arrangement determining. In any case, Gaussian processes are not restricted to regression they can likewise be stretched out to characterization and grouping errands. For a given arrangement of preparing focuses, there are possibly boundlessly numerous functions that fit the information. Gaussian processes offer an exquisite answer for this issue by doling out a likelihood to every one of these functions. The mean of this likelihood appropriation at that point speaks to the most plausible portrayal of the information. Besides, utilizing a probabilistic methodology permits us to fuse the certainty of the expectation into the regression result. Gaussian models are the basis for most old-style calculations for land spread order and target identification. A famous hyperspectral land spread characterization calculation is the quadratic 
discriminant analysis, otherwise called Gaussian greatest probability classifier or simply most extreme probability classifier. It is a discriminative model where the class contingent dissemination of the information is thought to be portrayed by a multivariate Gaussian dispersion, with the mean vectors and the covariance grids evaluated utilizing most extreme probability ${ }^{[5]}$. An exceptional situation where the entirety of the class covariance grids are thought to be the equivalent is called linear discriminant analysis. Gaussian models have additionally been broadly utilized in hyperspectral peculiarity and target location. The Mahalanobis separation indicator models the pixel estimations of a hyperspectral image utilizing a multivariate Gaussian, and names the pixels having low probability under this dispersion as peculiarities. The ReedXiaoli (RX) identifier broadens this by displaying just the area around the test pixel by a Gaussian circulation, not the whole image. Basic objective location calculations, for example, spectral coordinated channel and versatile cosine finder, additionally accept Gaussian conveyances for the objective and the foundation pixels. Gaussian models can likewise be found as parts in further developed calculations. For example, the grouping strategy by Persello et al., which performs both dynamic learning and move learning, uses Gaussian models. In this technique, the class probabilities of the information were demonstrated by Gaussian appropriations and question functions characterized over the class probabilities were utilized to iteratively evacuate models having a place with the source dataset from the training set and add models having a place with the objective dataset to the training set.

The paper $^{[6]}$ suggested that a significant utilization of airborne-and satellite-based hyperspectral imaging is the planning of the spatial dissemination of vegetation biophysical and biochemical boundaries in a situation. Measurable models, for example, Gaussian processes, have been fruitful for displaying vegetation boundaries from caught spectra, anyway their exhibition is exceptionally reliant on the measure of accessible ground truth. This is an issue since it is commonly costly to acquire ground truth data because of challenges and expenses related with test assortment and analysis. In this paper, we present two Gaussian processes-based methodologies for improving the precision of vegetation boundary recovery when ground truth is constrained. The first is the reception of 
covariance functions dependent on entrenched measurements, for example, spectral point and spectral connection, which are known to be better proportions of likeness for spectral information attributable to their strength to spectral inconstancies. The second is the joint demonstrating of related vegetation boundaries by perform various tasks Gaussian processes so the expectation precision of the vegetation boundary of intrigue can be improved with the guide of related vegetation boundaries for which a bigger arrangement of ground truth is accessible. We tentatively show the viability of the proposed strategies against existing methodologies on three genuine world hyperspectral datasets and one manufactured dataset. This paper recommended that the Hyperspectral sensors empower the investigation of the chemical and physical properties of scene materials remotely with the end goal of recognizable proof, recognition, synthetic structure analysis, and physical boundary estimation of articles in the earth. Consequently, hyperspectral images caught from earth watching satellites and airplane have been progressively significant in agribusiness, natural checking, urban arranging, mining, and protection. Machine learning calculations because of their exceptional prescient force have become a key device for present day hyperspectral image analysis. Along these lines, a strong comprehension of machine learning strategies have gotten fundamental for remote sensing analysts and professionals. The paper ${ }^{[7]}$ overviews and thinks about late machine learning-based hyperspectral image analysis strategies distributed in writing. We compose the strategies by the image analysis task and by the sort of machine learning calculation and present a two-route planning between the image analysis undertakings and the kinds of machine learning calculations that can be concerned them. The paper [8] is exhaustive in inclusion of both hyperspectral image analysis errands and machine learning calculations. The image analysis errands considered are land spread order, target/oddity discovery, unblending, and physical/compound boundary estimation. The machine learning calculations secured are Gaussian models, linear regression, strategic regression, bolster vector machines, Gaussian blend model, dormant linear models, meager linear models, Gaussian blend models, group learning, coordinated graphical models, undirected graphical models, bunching, Gaussian processes, Dirichlet processes, and deep learning. We additionally examine the open difficulties in the field of hyperspectral 
image analysis and investigate conceivable future bearings ${ }^{[9][10] .}$

Hyperspectral imaging (HSI) is a method that analyses a wide spectrum of light rather than simply doling out essential hues (red, green, blue) to every pixel. The light striking every pixel is separated into a wide range of spectral groups so as to give more data on what is imaged. The calculations and the image processing systems related with HSI are a result of military examination and were principally used to recognize targets and different items against foundation mess. Before, HSI has seen common applications, and has especially been valuable in satellite innovation. It may turn into a modest, promising, and snappy device for the appraisal of tissue conditions at finding and during medical procedure. The clinical applications incorporate crime scene investigation, identification of colorectal and gastric disease or ulcers ${ }^{[11]}$.

\section{Proposed Methodology}

Light hotspot for spectral imaging applications can for the most part be grouped into two classes: illumination and excitation source. Broadband lights are commonly utilized as the illumination hotspots for reflectance and conveyance imaging while narrowband lights are usually utilized as the excitation sources. In this way, illumination is a significant piece of the hyperspectral imaging framework. Contrasted and the unaided eyes, vision frameworks are influenced by the level and nature of illumination. Illumination gadgets produce light that enlightens the reviewed target objects; consequently, the presentation of the illumination framework can extraordinarily impact the nature of images and assumes a significant job in the general proficiency and precision of the framework. Great illumination can assist with improving the achievement of the image processing and analysis by diminishing commotion, shadow, reflection, and upgrading image differentiate. What's more, the positions, sorts of lights, and shading nature of the illumination are totally viewed as while picking the most appropriate illumination. Radiant lights, fluorescent lights, lasers, and infrared lights are the usually utilized light sources. The below block diagram shows that the information a hyperspectral cubical image is utilized. The information is separated into test information and training information. The training information will be processed by the Gaussian process and the normal framework will be 


\section{Advanced Multidisciplinary Scientific Research (IJAMSR) ISSN:2581-4281}

gotten from it. In view of the correlation between real test information and processed yield information the image classifier will produce the yield which is without blunder. Our methodology recoups the spectral subtleties from RGB images by inducing numerous arrangements of Gaussian Processes utilizing applicable hyperspectral training information and fastidiously joining them with the RGB images. We abuse the Kernels of Gaussian Processes to display the overall perfection of normally happening spectra. Additionally, the methodology likewise represents the non-cynicism of reflectance esteems and the self-comparability of hyperspectral image patches for precise spectral recuperation. The schematics of the proposed approach is delineated in Fig. 2. The general methodology can be isolated into training and testing stages. We give subtleties on every one of these stages' underneath. At the core of the training stage is learning of Gaussian Processes (GPi $\subseteq \mathrm{R} \mathrm{L}, \forall \mathrm{i})$ that speak to hyperspectral marks under a Bayesian model. For lucidity, we concede the subtleties on the proposed model and its surmising plan to the Sections to follow, and first sum up the rest of the training stage. The methodology pre-processes the training hyperspectral information by extricating $\mathrm{p} \times \mathrm{p} \times \mathrm{L}$ dimensional patches from the images and orchestrating them in ' $\mathrm{C}$ ' bunches utilizing $\mathrm{K}$ Means calculation. Processing hyperspectral images as far as grouped patches is worthwhile for our methodology for two principle reasons. 
Vol 3, Issue 10, 2020 Imfact Factor:2.58 DOI: https://doi.org/10.31426/ijamsr.2020.3.10.3811

\section{International Journal of Advanced Multidisciplinary Scientific Research (IJAMSR) ISSN:2581-4281}

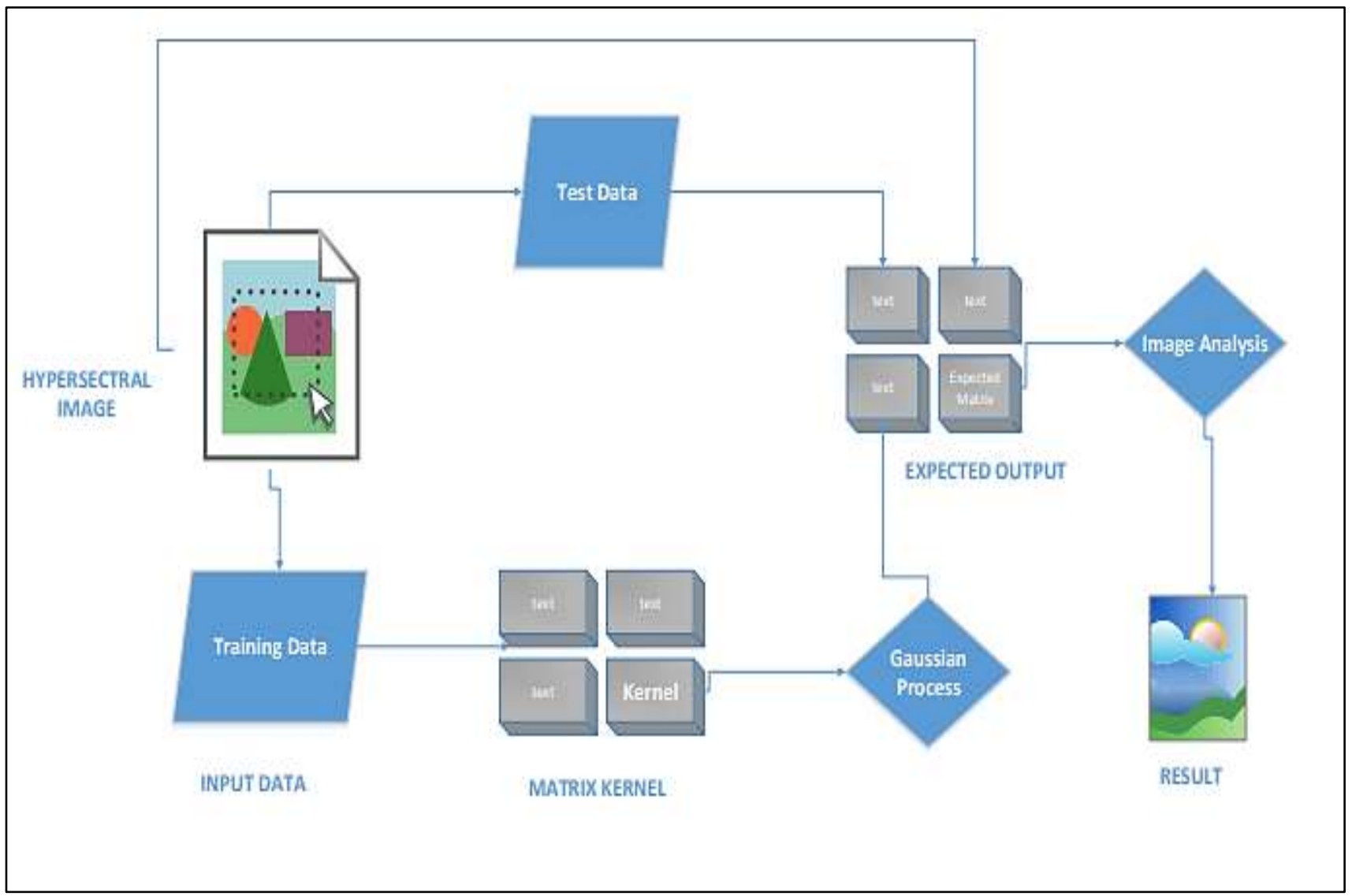

Figure 2 Hyperspectral Image Analysis

Initially, because of their similitude, the signs in each bunch are likely restricted to an even lower dimensional complex inside the sign space. Furthermore, inside each bunch the recurrence of the metameric is normally diminished in light of the fact that the groups are commonly made out of the equivalent/comparative spectral sources (for example patches comparing to 'grass' bunch well together). We realize that these properties of spectral marks are favourable for the exact recuperation of the spectral subtleties from
RGB images, which benefits the general methodology. We surmise a set $\mathrm{Gc}=\{\mathrm{GP} 1$, $\mathrm{GP} 2, \mathrm{GPK}\} \subseteq \mathrm{R} L$ of 'K' Gaussian Processes to speak to the ' $c$ ' bunch of the training information. As per our model, the Gaussian Processes mean the spectral sign sources in the training information. Henceforth, we insert the perfection of spectral marks into these Processes utilizing their Kernels. Additionally, we likewise utilize positive Mean priors in our model to fuse non-pessimism of reflectance esteems and the extents in which the spectral 


\section{International Journal of}

Advanced Multidisciplinary Scientific Research (IJAMSR) ISSN:2581-4281

signals by and large blend to frame image pixels. With the assistance of the Bayesian induction over the proposed model we learn back circulations for the Gaussian Processes. The Mean boundaries of the educated circulations are then changed to coordinate the spectral quantization of the RGB camera1.The changed boundaries for the "c" group as a network $\Phi$ e $\mathrm{c}=[\mu \mathrm{e} 1, \mu \mathrm{e} 2 \ldots, \mu \mathrm{eK}] \in \mathrm{R} 1 \times \mathrm{K}$, where $\mu \mathrm{ek} \in \mathrm{R} 1$ is the changed Mean of the" k" Gaussian Process for the bunch. This grid is later utilized in the testing period of the methodology. As portrayed before, the intensity of Gaussian processes lies in the decision of the kernel function. This property permits specialists to bring space information into the process and loans Gaussian processes their adaptability to catch patterns in the training information. For instance, by picking a reasonable data transfer capacity for the RBF kernel, we can control how smooth the subsequent function will be. A major advantage that kernels give is that they can be consolidated together, bringing about a more specific kernel. The choice which kernel to utilize is profoundly subject to earlier information about the information, for example in the event that specific qualities are normal. Models for this would be fixed nature, or worldwide patterns and examples. Fixed implies that a kernel is interpretation invariant and, in this way, not subject to the record ii. This additionally implies we can't demonstrate worldwide patterns utilizing a carefully fixed kernel. Recall that the covariance network of Gaussian processes must be sure semi-positive. While picking the ideal kernel blends, all strategies that save this property are permitted.

\section{Experimental Results}

In the figure beneath, the watched training information has a rising pattern with an occasional deviation. Utilizing just a linear kernel, we can impersonate a typical linear regression of the focuses. From the start, the RBF kernel precisely approximates the focuses. Be that as it may, since the Radial Basis Function RBF kernel is fixed it will consistently come back to $\backslash \mathrm{mu}=0 \mu=0$ in districts further away from watched training information. This reduction the exactness for expectations that venture further into the past or what's to come. An improved model can be made by joining the individual kernels through expansion, which keeps up both the occasional nature and the worldwide rising pattern of the information. This system can be utilized, for instance, in the analysis of climate information. The hyperspectral imaging is a helpful 
Vol 3, Issue 10, 2020 Imfact Factor:2.58 DOI: https://doi.org/10.31426/ijamsr.2020.3.10.3811

instrument to get and record the crude hyperspectral data of products of the soil. Be that as it may, because of the distinctions in camera quantum and physical design of imaging frameworks, the uncorrected brilliance for the various frameworks, in any event, for a similar framework utilized in various occasions, may be altogether different for a similar example taken under a similar condition. Consequently, precise calibrations for a hyperspectral imaging framework are important to ensure the soundness and agreeableness of the removed hyperspectral image information and the reliable execution of the framework. The first hyperspectral images can be aligned into the reflectance mode dependent on highly contrasting reference images.

\section{Radial basis function RBF \& periodic \& linear kernel outputs.}

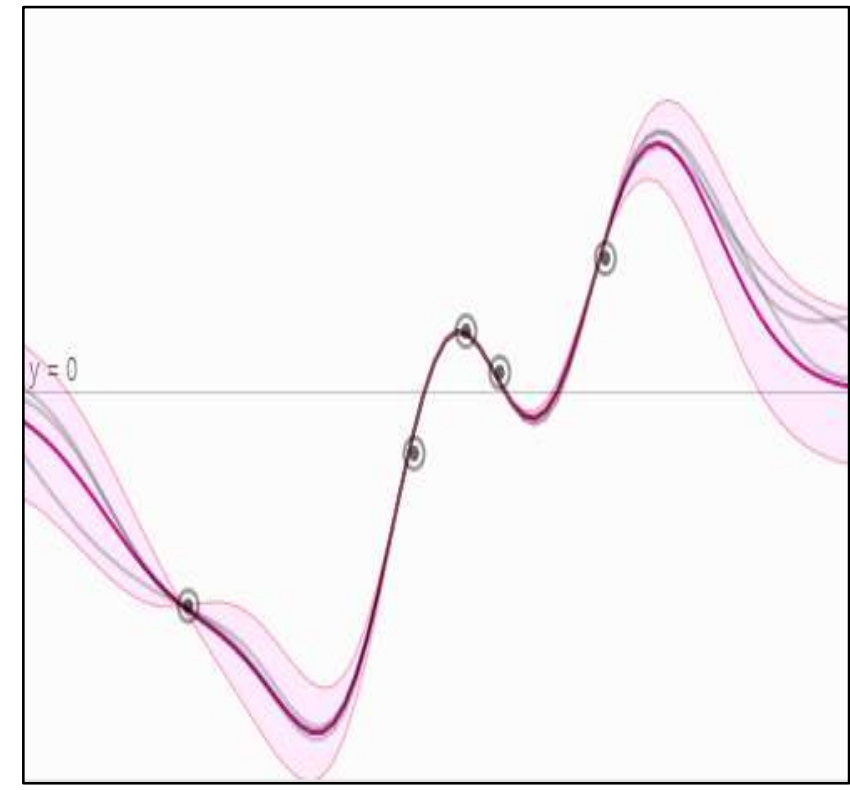

Figure 3 HSI INPUT 
Gaussian process can display questionable perceptions. This can be seen when just choosing the linear kernel, as it permits us to perform linear regression regardless of whether multiple focuses have been observed, and not all functions need to go legitimately through the observed training data.

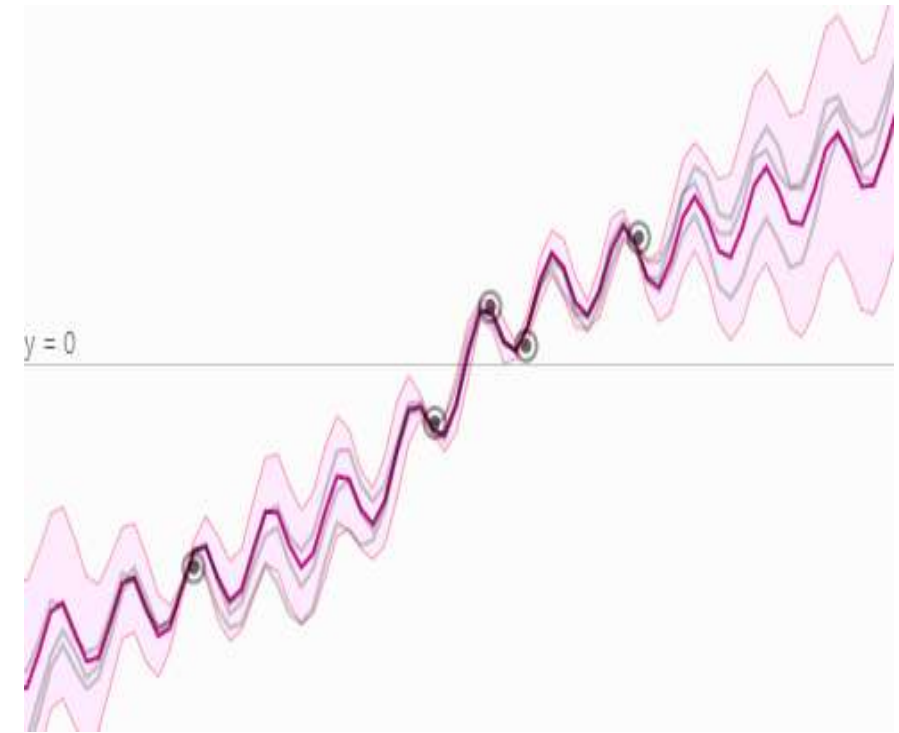

Figure 5 HSI INPUT 


\section{International Journal of Advanced Multidisciplinary Scientific Research (IJAMSR) ISSN:2581-4281}

Figure 6 GP OUTPUT

\section{Conclusion}

As we have seen, Gaussian processes offer an adaptable system for regression and a few augmentations exist that make them much more flexible. For example, in some cases it probably won't be conceivable to portray the kernel in basic terms. To overcome this test, learning particular kernel functions from the basic information, for instance by utilizing deep learning is a region of continuous exploration. Besides, connection between Bayesian inferring, Gaussian processes and deep learning will be investigated more in future papers. In this work, we proposed a method to computationally evaluate the hyperspectral image of a scene from its RGB image of known spectral quantization. The proposed approach accepts a hyperspectral training earlier over the imaged scene and concentrates groups of comparable patches from the training images. These bunches are utilized for figuring different arrangements of Gaussian Processes with the end goal that each Process connotes a spectral sign source in a group. The physical traits of the spectral marks are consolidated in the Gaussian Processes by their Kernels and the utilization of nonnegative Mean earlier likelihood appropriations. Even though we generally talk about Gaussian processes with regards to regression, they can be adjusted for various 
Vol 3, Issue 10, 2020 Imfact Factor:2.58 DOI: https://doi.org/10.31426/ijamsr.2020.3.10.3811

purposes, for example model-stripping and speculation testing. By looking at changed kernels on the dataset, domain experts or specialists can present extra information through proper mix and definition of the kernel.

\section{References}

1) Armin Schneider, Hubertus Feussner, in Biomedical Engineering in Gastrointestinal Surgery, 2017. "Hyperspectral Imaging".

2) Ni, C.; Wang, D.; Tao, Y. Variable weighted convolutional neural network for the nitrogen content quantization of Masson pine seedling leaves with near-infrared spectroscopy. Spectrochim. Acta Part A Mol. Biomol. Spectrosc. 2019, 209, 32-39.

3) Utsav B. Gewali 1, *, Sildomar T. Monteiro 2 and Eli Saber 1,3.2019 "Gaussian Processes for Vegetation Parameter Estimation from Hyperspectral Data with Limited Ground Truth". Remote Sens. 2019, 11, 1614; doi: 10.3390/rs11131614.

4) Zhang, X.; Lin, T.; Xu, J.; Luo, X.; Ying, Y. DeepSpectra: An end-to-end deep learning approach for quantitative spectral analysis. Anal. Chim. Acta 2019, 1058, 48-57.

5) Utsav B. Gewali1, *, Sildomar T. Monteiro2, and Eli Saber1,3. "Machine learning based hyperspectral image analysis: A survey". arXiv:1802.08701v2 [cs.CV] 10 Feb 2019.

6) ROSIS University of Pavia image. Obtained from GRSS DASE Website (http://dase. ticinumaerospace.com/). Accessed: 06-202018.

7) Chein-I Chang. A review of virtual dimensionality for hyperspectral imagery. IEEE Journal of Selected Topics in Applied Earth Observations and Remote Sensing, 2018.

8) Utsav B Gewali and Sildomar T Monteiro. A tutorial on modelling and inference in undirected graphical models for hyperspectral image analysis. International Journal of Remote Sensing, pages 1-40, 2018.

9) Remes, S.; Heinonen, M.; Kaski, S. Nonstationary spectral kernels. In Advances in Neural Information Processing Systems; The MIT Press: Cambridge, MA, USA, 2017; pp. 4642-4651. Available online: https: //papers.nips.cc/paper/7050-non-stationaryspectral-kernels (accessed on 8 May 2019).

10) Remes, S.; Heinonen, M.; Kaski, S. Nonstationary spectral kernels. In Advances in Neural Information Processing Systems; The MIT Press: Cambridge, MA, USA, 2017; pp. 4642-4651. Available online: https: //papers.nips.cc/paper/7050-non-stationaryspectral-kernels (accessed on 8 May 2019).

11) Zorzi, M.; Chiuso, A. The harmonic analysis of kernel functions. Automatica 2018, 94, 125-137, doi:10.1016/j.automatica.2018.04.015 